\title{
Methodology of analyzing the CdSe semiconductor quantum dots parameters
}

\author{
A. I. Mikhailov, V. F. Kabanov, E. G. Glukhovskoy, M. I. Shishkin, M. V. Gavrikov \\ Saratov State University, Department of Nanoand Biomedical Technologies, \\ Astrakhanskaya 83, Saratov, 410012, Russia \\ glukhovskoy@gmail.com
}

PACS 73.22.Dj, 73.21.La

DOI 10.17586/2220-8054-2018-9-4-464-467

\begin{abstract}
Direct methods (using a laser particle size analyzer) and indirect (from the analysis of spectral characteristics and differential normalized tunnel CVC) methods of CdSe QD size estimation allowed determination of the size $(4-5 \mathrm{~nm})$ and shown good qualitative and quantitative agreement of the results with an error of less than $10 \%$. It is concluded that the tunnel differential CVC analysis is an effective method for express measurement that can be used in quantum-size object investigations.
\end{abstract}

Keywords: quantum dots, cadmium selenide, differential tunneling current-voltage characteristics, energy spectrum.

Received: 5 February 2018

Revised: 14 June 2018

\section{Introduction}

Semiconductor structures, used in solid-state electronic devices, can contain single or multiple and associated quantum-size objects. Quantum dots (QD) are one of the most interesting quantum-size structures in terms of application in optoelectronics and nanoelectronics.

Semiconductor compounds $\mathrm{A}^{2} \mathrm{~B}^{6}$ are among the most promising and interesting semiconductor materials from a practical point of view, and have been so for many years. These materials have a high luminescence quantum yield, photostability and are widely used in optoelectronics [1-4].

The purpose of this paper is to develop and substantiate a methodology for studying the electrophysical properties of a monolayer film structure of semiconductor quantum dots of the $\mathrm{A}^{2} \mathrm{~B}^{6}$ group (e.g. CdSe).

\section{Samples obtaining technologies}

CdSe quantum dots monolayers, formed on the water surface and transferred to solid substrates with an indium-tin oxide (ITO) conductive layer by using Langmuir-Blodgett technology, were investigated. The quantum dots were synthesized according to previously-described methodologies [5-7].

\section{Research methods}

The obtained samples were investigated by scanning tunneling microscopy (STM), laser particle size analyzer and optical spectroscopy methods.

In the optical spectroscopy method, a standard violet LED with $500 \mathrm{mCd}$ luminous intensity and a spectrum maximum wavelength $380 \mathrm{~nm}$ was used as the primary excitation light source. Light from this source fell on a sample that was placed in a diffuse reflection attachment $\left(0-45^{\circ}\right)$, which allowed collection of light that was diffusely reflected from the sample and emitted by the sample (secondary). Then, the light passed through a band-pass filter, and in the long-wavelength part of the spectrum, a filter with a $360-600 \mathrm{~nm}$ transmission was automatically replaced by filter with a $600-1000 \mathrm{~nm}$ transmission. The measurement process was carried out in an automatic mode, the rate of the spectrum acquisition was $70 \mathrm{~nm} / \mathrm{min}$, and the sampling interval was $1 \mathrm{~nm}$.

Scanning electron microscope (SEM) and optical spectroscopy methods may not always provide unambiguous interpretation of the experimental data, e.g. in situations when the quantum-size particle has an inhomogeneous structure or when the several particles form a conglomerate. In this case, for more complete analysis of the electrophysical properties of the obtained QD film samples, in particular, the electronic spectrum, we used STM. This method was realized by using a scanning probe microscope NANOEDUCATOR-2. Before the investigation of nanoparticles tunnel CVC, the film surface was scanned by STM methods in a constant current mode. After analysis of the obtained STM image of the sample's surface, 10 points on the surface were selected for the obtaining of CVC. At each point, not less than $10 \mathrm{CVC}$ were obtained in automatic mode. Measurements were carried out for current values ranging from $10^{-11}$ to $10^{-7} \mathrm{~A}$ and voltage ranging from 0 to $5 \mathrm{~V}$. By reproducibility of the measurement results, we selected points with stable characteristics, and after that, we averaged the obtained 
CVC. The measurements results were processed according to the generally accepted methods for scanning probe microscopy of semiconductor nanostructures.

For the experimental tunnel CVC analysis, we used the differential CVC method - dependence $(d I / d V) /(I / V)$ on the voltage $V[8,9]$. Additionally, as shown in [10,11], this method can be used for analyzing the investigated structures' conductivity mechanisms, parameter calculation and a number of other important electronic processes.

\section{Model and numerical estimates}

The model of electrons tunneling through the energy levels of the discrete spectrum of the QD was considered in [12].

When QD model of a cubic shape with an edge a, the position of QD energy spectrum levels:

$$
\varepsilon_{i}=\frac{(\pi \hbar)^{2}}{2 m^{*}} \cdot \frac{1}{a^{2}} \cdot\left(l^{2}+m^{2}+n^{2}\right),
$$

where $l, m, n=1,2,3, \ldots$ - are positive natural numbers corresponding to the level numbers; $m^{*}$ - effective mass of the electron; $a$ - characteristic size of the QD.

In the case of a spherical QD with radius a in the one-electron spectrum in a parabolic well approximation:

$$
\varepsilon_{i}=\frac{(\pi \hbar)^{2}}{2 m^{*}} \cdot \frac{1}{a^{2}} \cdot(4 n+2 l+3),
$$

where $n$ - radial quantum number $(n=0,1,2, \ldots) ; l$ - orbital quantum number $(l=0,1,2, \ldots)$.

The calculated electron energy values for the first three allowed energy levels $(1,2,3)$ in accordance with (1), (2) for CdSe are shown in Fig. 1(b). The electron's effective mass value in the CdSe conduction band, used in calculations $m^{*}=0.13 m_{0}$, where $m_{0}$ - is the free electron mass. In accordance with this model it is possible to estimate the QD characteristic size.

We considered and analyzed normalized differential tunneling CVC with a negative bias potential on the substrate relative to the probe (Fig. 1a). In this case, electrons tunnel from the ITO electrode through the discrete levels of a quantum-size object to the tunneling microscope probe. The conduction electron discrete energy spectrum of a quantum-size object determines the peaks on the normalized differential CVC (arrows in Fig. 1). The black solid line in Fig. 1a is a trend line constructed using linear filtering (by MS Excel tools).

The obtained values on the axis of the bias potential were put in correspondence with the energy values on the calculated energy spectrum dependence for the models used. For example, in Fig. 1, the peak values corresponded to $0.40 \mathrm{~V} ; 0.62 \mathrm{~V} ; 0.81 \mathrm{~V}$. On the graphs of the energy spectra (Fig. 1b), this corresponds to a range of QD values of $4.4-4.8 \mathrm{~nm}$ for the "spherical" model and $4.3-5.4 \mathrm{~nm}$ for the "cubic" model.

Analysis of the peak's position on differential normalized tunnel CVC on the basis of averaging over a group of samples allowed us to estimate the linear size of quantum-size objects, which ranged from $4-5 \mathrm{~nm}$ (with an error in the peak's position measurement not more than $2 k T$ ). The use of a "spherical" QD model for the samples makes it possible to obtain more accurate size determination than the "cubic" QD model: the range of values on the "size" axis compared with the corresponding ranges of energy values is defined much more accurately (Fig. 1b).

To clarify the validity of this method based on the analysis of the peaks position on differential normalized tunnel CVC, in addition, we estimated the QD size by analyzing the spectral characteristics of the samples (Fig. 2). In Fig. 2, samples with different QD formation time (from 15 to $300 \mathrm{sec}$ ) are presented.

The QD size estimation, when considering Eqs. (1) and (2), the energy of the width of the material band gap $\varepsilon_{g}=1.74 \mathrm{eV}$, and assuming that the change in the position of the QD first energy level $\varepsilon_{c 1}$ relative to the conduction band bottom of bulk material $\varepsilon_{c}$ is several times greater than the corresponding change the valence band top energy $\varepsilon_{v 1}$ relatively $\varepsilon_{v}$ (because $m_{n}^{*} \ll m_{p}^{*}$ ), gave the result $4.1-5.1 \mathrm{~nm}$.

The results of CdSe QD investigations using the Malvern ZetaSizer Nano ZS laser particle size analyzer are shown in Fig. 3.

The obtained results, with an assumed QD shell thickness of $\sim 1 \mathrm{~nm}$, showed the characteristic QD size of $3.9-4.9 \mathrm{~nm}$.

The processing of the investigated samples made it possible to conclude that the calculated QD sizes obtained by experimental differential tunnel CVC analysis, spectral characteristic analysis and measurement with a laser particle size analyzer are in good qualitative and quantitative agreement. 


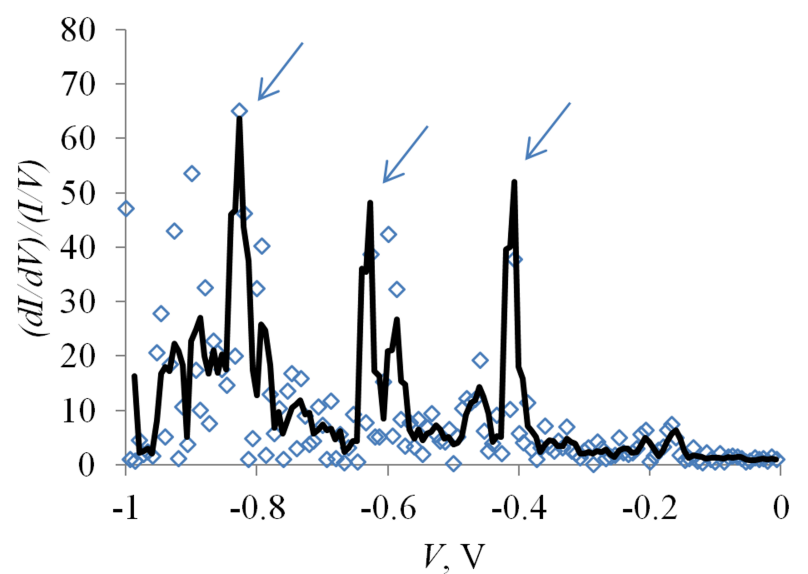

(a)
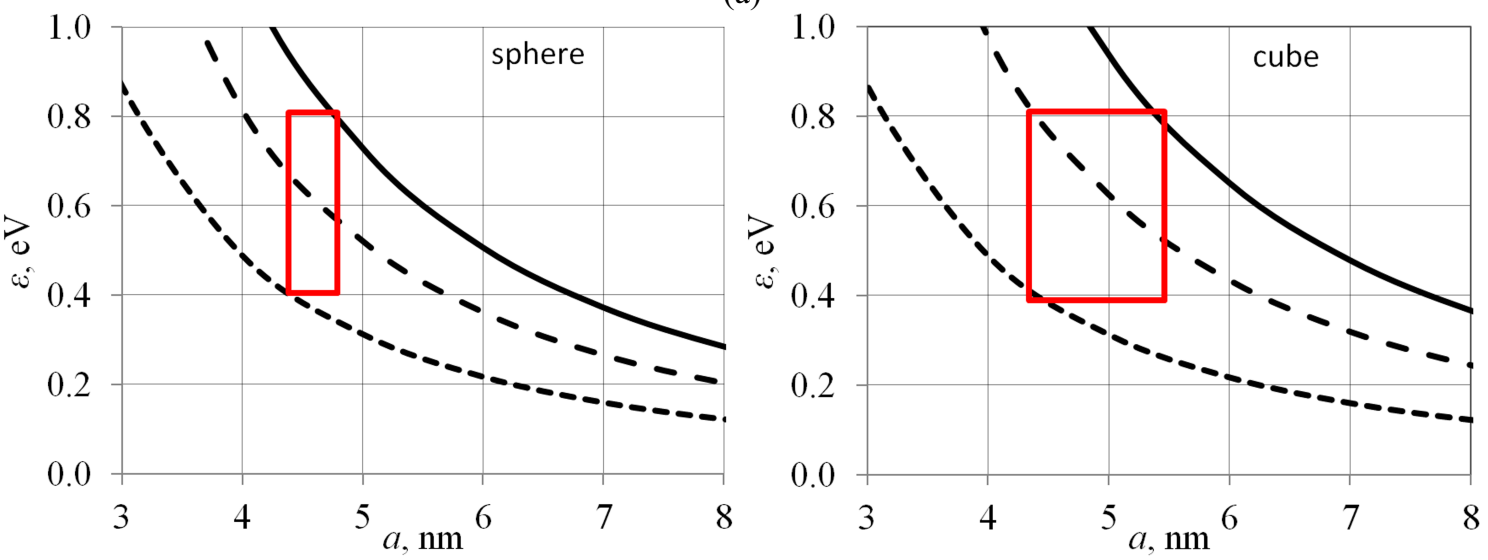

(b)

FIG. 1. Typical differential current-voltage characteristics of CdSe QD (a), (b) - accordance with the calculated levels of the energy spectrum according to the spherical QD model and according to the cubic QD model

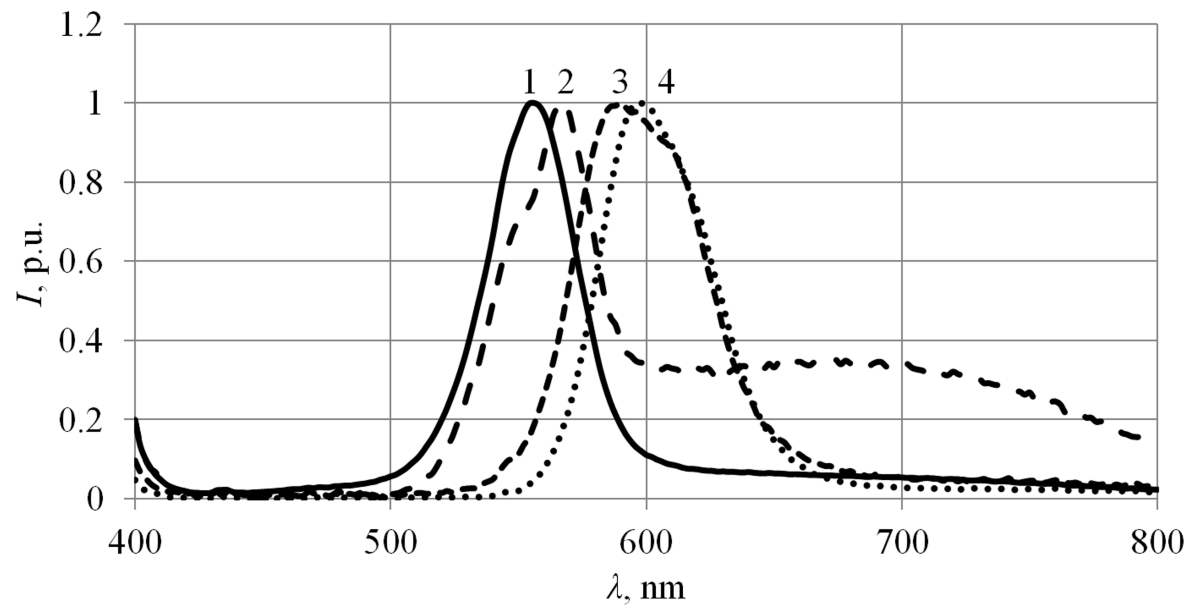

FIG. 2. Spectral characteristics of CdSe QD: 1, 2, 3, 4- different samples 


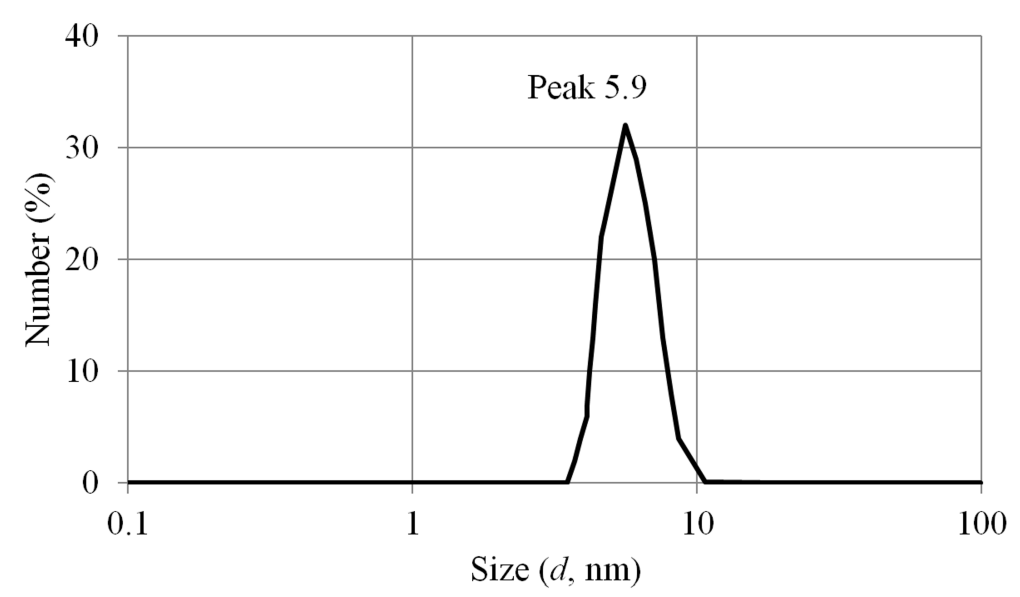

FIG. 3. CdSe QD size estimating using a laser particle size analyzer

\section{Conclusions}

Thus, based on the CdSe QD size estimate analysis, we can conclude that both direct (using a laser particle size analyzer) and indirect (from the analysis of spectral characteristics and differential normalized tunnel CVC) methods of QD size determination have shown good qualitative and quantitative agreement of the results with an error less than $10 \%$. Additionally, the differential normalized tunnel CVC analysis method made it possible to determine a more suitable model for determining the energy spectrum of the investigated samples - the spherical QD model. Moreover, this method can be used to analyze the conductivity mechanisms of investigated structures, as well as calculate parameters for many other important electronic processes. All this allows us to consider, that the differential normalized tunnel CVC method is an effective method for rapid quantification, which can be used in studies involving quantum-size objects.

\section{Acknowledgements}

The authors are sincerely grateful to Prof. I. Goryacheva for provided samples of quantum dots. This work was supported by grants from the Russian Foundation for Basic Research Projects No. 16-07-00093 and No. 16-07-00185.

\section{References}

[1] Karpovich I.A. Quantum engineering: self-assembled quantum dots. Soros educational journal, 2001,7 (11), P. 102-108 (in Russian).

[2] Karpov S.V., Mikushev S.V. Electron-hole excitations in CdSe quantum dots under strong and intermediate confinement conditions. Physics of the Solid State, 2010, 52 (8), P. 1750-1756.

[3] Boichuk V.I., Leshko. R.Ya., Holskyi V.B., Karpyn D.S. Optical spectra of small CdS nanocrystals. Semiconductor Physics, Quantum Electronics \& Optoelectronics, 2016, 19 (4), P. 384-390.

[4] Vitukhnovskii A.G., Vashchenko, A.A., Lebedev V.S., et al. Organic light-emitting diode with an emitter based on a planar layer of CdSe semiconductor nanoplatelets. JETP Letters, 2014, 100 (2), P. 86-90.

[5] Reiss P., Protiere M., Li L. Core/shell semiconductor nanocrystals. Small, 2009, 5 (2), P. 154-168.

[6] Speranskaya E.S., Goftman V.V., Goryacheva I.Yu. Preparation of water soluble zinc-blende CdSe/ZnS quantum dots. Nanotechnologies in Russia, 2013, 8 (1-2), P. 129-135.

[7] Kosolapova K.I., Al-Alwani A.J.K., Gorbachev I.A., Glukhovskoy E.G. Purification non-aqueous solution of quantum dots CdSe-CdS-ZnS from excess organic substance-stabilizer by use PE-HD membrane. J. Phys.: Conf. Ser., 2015, 643, P. 012084(1-5).

[8] Troyan V.I., Pushkin M.A., Borman V.D., Tronin V.N. Physical basis of techniques for studying nanostructures and surface of solids, Ed. by V.D. Borman, MEPhI, Moscow, 2008, 260 p. (in Russian).

[9] Demikhovskii V.Ya., Filatov D.O. Scanning probe microscopy study of electronic states in low-dimensional structures: learning guide on physicochemical fundamentals of nanotechnology, Nizhny Novgorod, 2007, 77 p. (in Russian).

[10] Mikhailov A.I., Kabanov V.F., Zhukov N.D. Peculiarities of field electron emission from submicron protrusions on a rough InSb surface. Technical Physics Letters, 2015, 41 (11), P. 1065-1067.

[11] Mikhailov A.I., Kabanov V.F., et al. Electronic properties of A2B6 quantum dots incorporated into Langmuir-Blodgett films. Bulletin of the Russian Academy of Sciences: Physics, 2017, 81 (12), P. 1472-1475.

[12] Mikhailov A.I., Kabanov V.F., Zhukov N.D., Glukhovskoy E.G. Features of the energy spectrum of quantum dots indium antimonide. Nanosystems: Physics, Chemistry, Mathematics, 2017, 8 (5), P. 596-599. 